\title{
Data Mining for Adivasi Vikas Vibhag Mobile App Usage Predictions and Recommendations
}

\author{
Madhavi Khode-Chaware ${ }^{1}$ and Aarti Karandikar ${ }^{*}$ \\ ${ }^{1}$ Ex. Additional Commissioner, Tribal Development, Nagpur, India \\ ${ }^{* 2}$ Assistant Professor, Shri Ramdeobaba College of Engineering and Management Nagpur, India
}

\section{ABSTRACT}

The main purpose of digital technology is to connect every strata of the society. The utilization of technology by means of 'mobile government' or 'm-government'can be used to bridge the gap between public needs and services that the government provides. One form of the 'm-government' is the usage of mobile application (mobile app) as a medium of communication.Tribals or Adivasis as they are referred in India are one of the less connected and cut off communities from mainstream. Due to many geographical and technical reasons, both sides - the adivasis and the administration - find it difficult to mingle with each other. At the same time taking every policy to their door step is financially and practically a daunting task as they live in far remote places and hence the communication gap. This paper is a study of the "Adivasi Vikas Vibhag" Mobile App which is one such step towards bridging the communication gap between tribal and administration on digital platform. The data collected through this app has tremendous potential for application of data science tools. Mobile data science is a fast emerging field that involves collecting the mobile phone data from various sources and building data-driven models using machine learning techniques, which in turn will help in making dynamic decisions intelligently in various day-to-day situations of the users.In this paper we discuss how mobile data science can be applied to data collected through Adivasi Vikas Vibhag Mobile App to make intelligent decisions and to enhance user experience.

KEY WORDS: ADIVASI VIKAS, TRIBAL DEVELOPMENT, GOVERNMENT SCHEMES FOR ADIVASIS, DATA SCIENCE.

\section{INTRODUCTION}

Adivasis are anintegral part of India and account for $8.2 \%$ of India's population. These communities are found all over the states and union territories of India. Due to their different lifestyle and different ideology, culture, tradition and social values they are cut off from mainstream society. The solution to most of the problems lie in keeping tribals informed about various schemes of

\section{ARTICLE INFORMATION}

Received 19th Oct 2020 Accepted after revision 28th Dec 2020 Print ISSN: 0974-6455 Online ISSN: 2321-4007 CODEN: BBRCBA

Thomson Reuters ISI Web of Science Clarivate Analytics USA and Crossref Indexed Journal

\section{Clarivate
Analytics}

NAAS Journal Score 2020 (4.31)

A Society of Science and Nature Publication,

Bhopal India 2020. All rights reserved.

Online Contents Available at: http//www.bbrc.in/

Doi: http://dx.doi.org/10.21786/bbrc/13.14/25 their benefit.If we can reach out to these people and help them to grow at their traditional habitat, we will be able to preserve the culture and values of these communities at the same time it will help to preserve cultural and social heritage of India.In Maharashtra,there are 10.33 million tribals like Kolam, Katkari, Bhil, Gond/Rajgond, Warli, Kokana, Thakur, etc. There are many schemes and policiesby government for these tribals. But the ground reality is that government does not have machinery or a system where person to person contact with each Adivasi is technically possible.

A practical case of Adivasis from far remote place called as Timeli which is some $50 \mathrm{~km}$ from Bhamragarh will make thing more clear. Astonishingly, even Google map fails to show the road map and distance of this place. If any person from a small village from above example, like Timeli or similar remote small village wants to know 
some information about Government schemes, he has to spend a day's time and almost one day's wage of his monthly income just to get the information about the scheme of his interest in first visit. Then he goes back to his place, collects all documents and again spends another 3 to 4 days wages of his income in multiple visits to offices for submission of documents, verification and follow-ups. If the problem is not solved or the need is not fulfilled at Bhamragarh, he has to come to Gadchiroli or to Nagpur where he spends much of his monthly income to get the right information about particular scheme. Overall situation is such that he spends almost 20 to $25 \%$ of monthly income along with loss of almost 4 to 5 man days and a loss of equal amount of energy in successful filing of an application to get the benefits of Government schemes.

In such scenario, digital connect is the best possible way to reach out to every Adivasi in the shortest span. This is economically viable for both parties, administration as well as Adivasis.Developed under guidance of Additional Tribal Commissioner (ATC) Nagpur Dr. Madhavi KhodeChaware, and endorsed by Maharashtra Government, the Adivasi Vikar Vibhag mobile app is on its way to success. The app is getting good response from the tribals with over 23000 download in 1.5 years. This paper focuses on possible applicationsof mobile data science tools on the data collected through the existing app. A quantitative analysis of the data will revealwhat type of schemes are popular in a particular district or area, what type of problems tribals of a particular areaare commonly facing, what type of documents are frequently needed to apply for a given scheme, how manypeople benefited, which schemes are garnering good response, and factors influencing availing of various schemes. An analysis of the app surfing patterns of the adivasi can disclose future demands or needs of the tribals. Thiscan be used to create a predictive tool to study problems faced by tribals regarding various government schemesand policies and use it to evaluate different schemes and policies.

\section{MATERIAL AND METHODS}

This research uses an 'exploratory research' approach to explain how the mobile app is used in the context of providing vital information, about various schemes and policiesof the government, to the Adivasis.Exploratory research is the process of investigating a problem that has not been studied or thoroughly investigated in the past.

The information in app is classified in 5 different heads on the home page:

\section{Gallery}

2. Different Schemes of Government

3. Important Addresses and Contact Numbers

4. Suggestions/Requests/Enquiries/Queries.

5. Important Services for citizens

The most important Tab is Tab Number 2 which showcases different schemes of government. This Tab is further sub classified as:
1. Schemes of Educational Importance

2. Schemes for Individual Benefit

3. Schemes for regional development

4. Schemes for community development

5. Other Important Events.

Figure 1: App interface of Tab Number 2 showing different schemes of government.

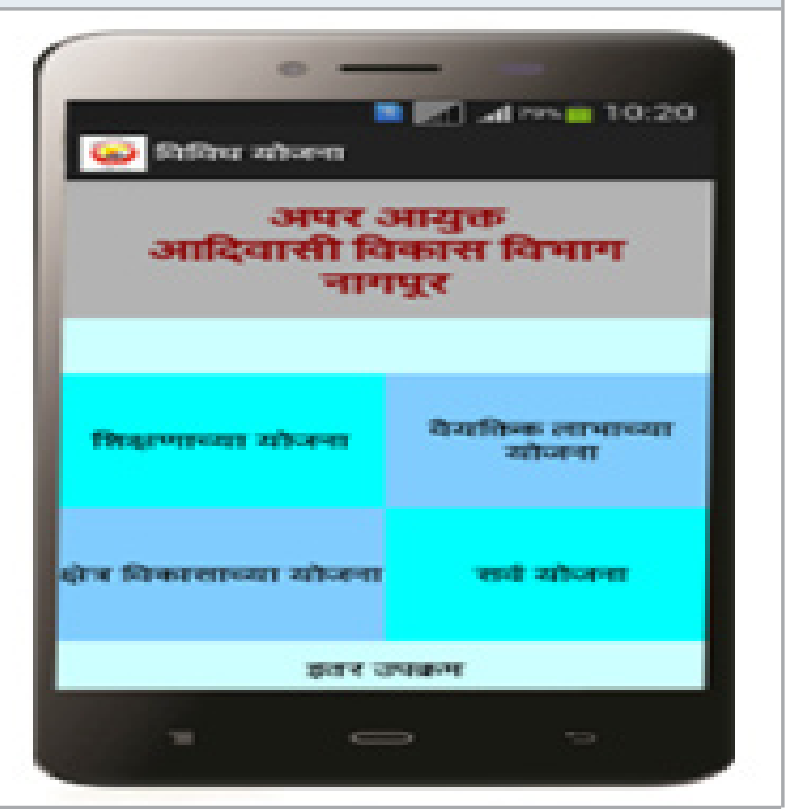

Every sub-tab is further divided into categories which are easy to understand. For example under Educational Schemes, the app provides 4 important categories:

1. Primary Education

2. Hostels for tribal students

3. Scholarships / and fee structures

4. Other educational schemes

Figure 2: Page showing different schemes under a category.

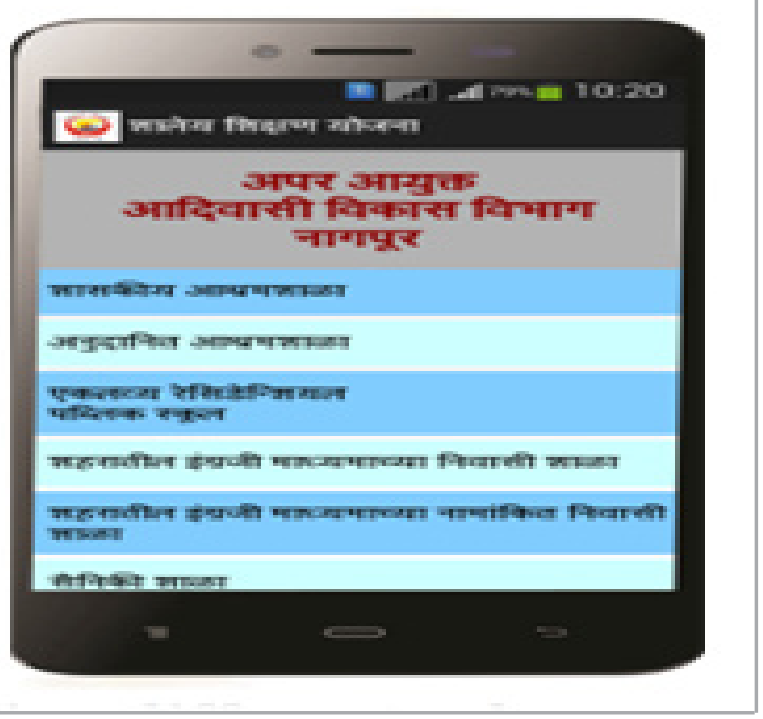


Once we select a category of our interest, the app provides full listing of all schemes of selected categories. The user can select any scheme of his/her interest and search all detail information of the scheme. The following important information is provided in Marathi language for the user that is Adivasi.

1. The outline of scheme.

2. Eligibility criteria

3. Necessary documents required for the scheme.

4. Sample form.

5. All important addresses and contact numbers of concern departments and officers.

\section{DISCUSSION}

This research uses sample data set for illustration purpose. To implement the algorithms of data science and to improve the throughput of the digital application from perspective of planning and implementation, it is very much necessary to collect the data in a scientific way. The scientific data collection is a first step towards implementation of effective data science algorithms. Consider the following descriptive statistics of the app are : there are around 23000 downloads of the app till September 2020 and the app is opened for 76000 times. This means on an average after downloading every user is visiting the app three or four times.Currently, the app does not support user registration. If this feature is included in the app, it will facilitate maintaining user related information. The users can be classified as 'traveller' or 'explorer' where traveller is a user who browses the app at a glance and explorer is a user who is interested in gathering information provided in the app. Number of hits for a traveller will be very less, hardly one or two, as compared to a explorer who may visit the app multiple time, usually three and more. If explorers are identified, the app can be reconfigured to cater to their specific needs.

Table 1. Number of hits on Tab Number 2 (Representative data only for illustration purpose)

\begin{tabular}{|l|c|}
\hline $\begin{array}{l}\text { Name of } \\
\text { the scheme }\end{array}$ & $\begin{array}{c}\text { Number } \\
\text { of hits }\end{array}$ \\
\hline Schemes related to education & 23001 \\
\hline Schemes related to individual benefits & 65780 \\
\hline Schemes for regional development & 52311 \\
\hline Schemes for community development & 38967 \\
\hline Other miscellaneousschemes & 16554 \\
\hline Total hits & 196613 \\
\hline
\end{tabular}

Table 1 shows representative data about the number of hits on Tab Number 2 which showcases different schemes of government. The figures suggest popularity of schemes of individual benefits by the Adivasis. Table 2 gives representative data about number of hits under various schemes of individual benefits.
Table 2. Number of hits for Individual Beneficiary schemes (Representative data only for illustration purpose)

\begin{tabular}{|l|c|}
\hline $\begin{array}{l}\text { Name of } \\
\text { the scheme }\end{array}$ & $\begin{array}{c}\text { Number } \\
\text { of hits }\end{array}$ \\
\hline Schemes for youths & 34988 \\
\hline Schemes for farmers & 26655 \\
\hline Schemes for women & 11970 \\
\hline Schemes for household & 22008 \\
\hline Other schemes for individual benefit & 15607 \\
\hline Total hits & 111228 \\
\hline
\end{tabular}

Using statistical analysis methods like Linear Regression we can predict a dependent variable by fitting a best linear relationship. Classification techniques of data mining can be used to categorize the users. In this app, the databasecan beredesigned to record the details of browsing pattern. Every page hit, event hit, session hit could be properly logged and stored in the database. Patterns like which types of schemes get maximum hits in which months, preference of specific section of users for specific schemes can be generated. Association mining can be applied to find which schemes are searched together.

If search patterns for a user are stored, layout of the screen can be dynamically generated based on his preferences. Natural language processing can be applied on the queries asked by the users and a Frequently Asked Questionnaire can be automatically generated. The automatic discovery of user navigation pattern can be done by tracking button/tab clicks. The button/tab click logs are created on daily basis at the time a user clicks a particular button/tab. The paper suggests restructuring of app contents according to the user preference and pattern. An optimal path for every type of user can be generated by considering the click pattern.

\section{CONCLUSION}

The data of app downloads for informative government apps show that the Adivasis and job seekers are more inclined towards digital platform for information collection than other class of people. Those who are struggling and those who want to explore future prospects use informative government apps more than those who are economically stable. This proves that the app is a best media to reach out to Adivasis. Hence we should develop more digital material for Tribals of India to provide equal opportunities of growth. Right from date of launching there is a steady and consistent download for the app. Almost 500 downloads per month is a rate of download. Considering the illiterate class with minimal connects and exposure to cyber world, we consider this rate as amazing response. This shows the thrust of every Indian to acquire the information of his/her importance. 
Authors felt that the app is an important milestone on the path of Digital India and will be instrumental in bridging the gap between urban and developed society on one side and Tribals or Adivasis on other side. Platforms like this provide an excellent opportunity for industry-institute collaborations. In future, user registration facility can be added in the app. The data can be stored on a cloud based platform for online registration, scrutiny and approval. Mobile data science tools and analysis methods can be applied on the data collected through this app to gain meaningful insights into the requirements of the Adivasis which will help the administration in making decisions and introducing new schemes.

\section{REFERENCES}

Barbaro, Eduardo et al. (2019) Modelling and Predicting User Engagement in Mobile Applications. Data Science, pp. 1-17.

Deepak Mangal, Saurabh Singhal, and Dilip Kumar Sharma (2019). An Algorithm for Prediction of Web User Navigation Pattern and Restructuring of Web Structure Based on Visitor's Web Access Pattern. Advances in Computing and Data Sciences, pp. 689-700,D0I: 10.1007/978-981-13-9942-8_64

Dey, S. (2012). Essay on the general characteristics of Tribal Economy in India. Preserve Article. Retrieved from http://www.preservearticles.com/essay-on-thegeneralcharacteristics-of-tribal-economy-in-india. html

Farhana Seemi et al. (2019) Browsing Behaviour Analysis using Data Mining. International Journal of Advanced Computer Science and Applications Vol 10(2), pp 490-498.

Girase, S. (2016).The Problems of Indian Tribal
Communities in Current Scinario. International Journal of Development Research. Vol. 06, 7924-7927

Hong Cao and Miao Lin (2017). Mining smartphone data for app usage prediction and recommendations: Asurvey. Pervasive and Mobile Computing, Elsevier, Volume 37, June 2017, Pages 1-22 https://doi. org/10.1016/j.pmcj.2017.01.007

Iqbal H. Sarker (2018). Mobile Data Science: Towards Understanding Data-Driven Intelligent Mobile Applications. EAI Endorsed Transactions on Scalable Information Systems 062018 - 122018 , Volume 5 , Issue 19, e4, pp 1-11.

Kushchu, I. \& Kuscu, M.H. (2003) From E-government to M-government: Facing the Inevitable. 3rd European Conference on e-Government, Ireland, pp. 253-260, 2003.

Munmun Sen (2018). Tribal Development: A New Vision for Transforming India. International Journal of Recent Scientific Research, ISSN: 0976-3031 Vol. 9, Issue, 12(E), pp.30118-30121.

Shendy M Adam Firdaus, Irwansyah, and K. Djaja (2016) Mobile apps as government communication media in urban public services: case study - the usage of Qlue application by Jakarta Provincial Government. WIT Transactions on Ecology and The Environment, Vol 210 ISSN 1743-3541 (on-line), pp 417-430

Socio-economic Development Of Scheduled Tribes. http://ncst.nic.in/content/socio-economicdevelopment Zhung-Xun Liao et al. (2013) On Mining Mobile Apps Usage Behavior for Predicting Apps Usage in Smartphones. In Proc. of CIKM, pp 609-618.

Disclaimer : The views, thoughts, and opinions expressed in the paper are those of the authors. 